\title{
Material Studies for Thermal Responsive Composite Envelopes
}

\author{
Isak Worre Foged ${ }^{1}$, Anke Pasold ${ }^{2}$, Tommaso Pelosini ${ }^{3}$ \\ ${ }^{1}$ Aalborg University ${ }^{2}$ Material Design Lab / Copenhagen School of Design and \\ Technology ${ }^{3}$ AREA \\ Iiwfo@create.aau.dk²ankp@kea.dk³tommaso@studio-area.net
}

\begin{abstract}
The material-based studies examine through computation and physical prototyping layered composites for thermal responsive building envelopes. Focus is placed on surveying and computing a large series of materials across four groups, for then to test these materials from factors of solar energy reception capacities, internal heating methods, heat isolation coatings and layer bonding. An oak-polyethylene structure is developed based on the first studies and further tested towards implementation as part of an adaptive envelope demonstrator, with these studies focused on fabrication and assembly methods. Results of the developed, tested and applied composite as part of an adaptive envelope shows that the environmental-material composite is strongly influenced by colour and direct solar radiation exposure. This in turn allow a material-fabrication approach to program a responsive system driven by exergy. Reinforcing the responsive reaction of the composite by internal heating does not advance the performance, as coatings are needed to maintain the heat inside the material, which adds weight and isolate the composite from the thermal environment that otherwise is intended to provide the energy for driving the responsive behaviour. Please write your abstract here by clicking this paragraph.
\end{abstract}

Keywords: Material Studies, Thermal Responsive, Composites, Building Envelopes

\section{INTRODUCTION}

This work and paper are focused on how materials can be combined into thermal responsive composites. Through the focus on material properties, assembly processes and thermal energy distribution in the composites, the study contributes to new understanding of the thermal reactive performance of layered materials. In this way, the work is intended as foundational research for intrinsic responsive and adaptive architectural surfaces, which are driven by material-environmental properties and interactions, in contrast to extrinsic mechanically actuated systems.

Previous research on material based responsive systems can be seen by thermodynamic (Foged and Pasold, 2015, 2016) and hydrodynamic (Correa et al 2013, Reichert et al, 2015, Raviv et al, 2014) studies, where both direct (material as actuator and en- 
vironmental element) and indirect (material as actuator of an environmental element) actuation is studied. For layered material responsive actuation, based on material differences in thermal expansion, studies (Foged and Pasold, 2010, 2015, 2016) have been conducted, and in industrial products the use of bimetals is developed and used (Kanthal, 2008) as an actuator, typically used as triggers for an intended temperature indication, such as a boiling point.

The background on potential solutions of this work is a data collection surveying of a large set of materials to understand through computed combinatorial analysis the material responsive performance of a multitude of resultant composites. Additionally, increased understanding of connectivity between layers is important to utilise the composite's responsive properties. Bonding studies with different adhesives of material layers and internal/external heat activation of layers are studied to understand both fabrication constraints and environmental and system activation of thermal-based responsive composites.

The paper presents firstly the survey and computed combinatorial analysis of approximately 200 materials, across 4 material groups. Following the categorization and understanding of materials through computed models, ten materials are selected for physical prototyping studies, where composites are fabricated to investigate the physical bending when exposed to actuation under gravity and thermal gradients. Additionally, the paper illustrates how a selected composite can be applied in an auxiliary adaptive envelope and installed as an onsite full-scale demonstrator. This effort points to the potentials of combining material-environmental responsive structures with mechanical-microprocessor driven mechanisms for coupled response properties. Lastly, a discussion of the material studies, their relevant integration as architectural responsive surfaces and future outlook is presented.

\section{METHODS}

The study uses a hybrid approach of material surveying, categorization, combinatorial analysis and physical prototyping. Each method is a stepwise delineation and filter the research of responsive performance based on composition of material layers.

\section{Material surveying}

Material surveying is done via online portals and, most notably, the Material Connexion Library in combination with Granta's CES Edupack. Two principal values are extracted, namely the thermal expansion coefficient, alpha, (10-6 m/(m K)), determining the amount of expansion in relation to temperature change, and Young's Modulus, E, (GPa), determining the elasticity of a material. 193 materials are listed across wood species, plastics, metals and (other than wood) organic materials. Additional information is gathered for availability/rarity, sub-types of colours, densities et cetera. A similar survey is made of bonding materials, including acrylics, adhesive, liquid sealants, lockers and structural glues are included. The primary parameter assessed is the shear modulus, $\mathrm{G},(\mathrm{N} / \mathrm{mm} 2)$, as the shear force between layers may lead to delamination during asymmetric expansion of the composite layers.

\section{Combinatorial Analysis Studies}

Based on an ordered table of materials and their specific properties, a computed combinatorial analysis is made in MS Excel, computing all possible pairlayering combinations of the materials, resulting in bending properties, deflection from a plane $(\mathrm{mm})$ and a graph representation, which visualise the resultant bending of the specific composite, figure 1 . This is done by using the industry formula (Kanthal, 2008) for bi-metals, where in this case, geometric and temperature variables are constant, hence, the relative performance of material composites measured against each other. 
Figure 1

Deflection of composite

materials are

calculated as

bi-materials, but

consist in reality of

more layers,

including 1-2

binding layers,

which effect the

properties of the

composite structure

in terms of

strength, elasticity

and thermal

conductivity. The

responsive bending

can be positive and

negative in

direction

depending on layer

composition.

Figure 2

Graph of surface

temperatures as a

result of solar

radiation and

material properties.

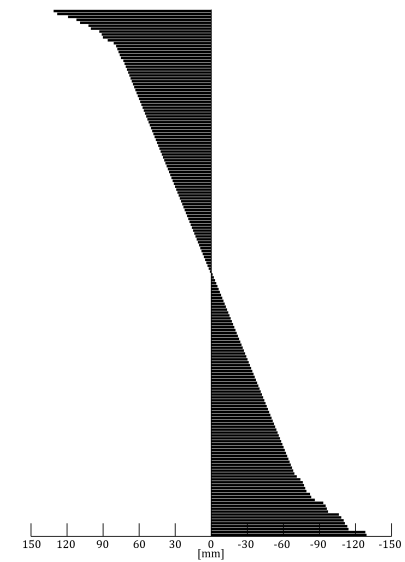

\section{Prototyping Studies}

Ten materials are chosen for physical prototyping studies, including Oak, Walnut, Pine, Polypropylene (PP), Polyethylene (PE), Polyvinylchloride (PVC), Copper, Aluminium, Bronze and Styrofoam. The materials are then combined to study the adhesive properties between low and high energy surfaces, their response to heat change and how heat can be induced between layers to increase responsive performance beyond the temperature change created by microclimatic changes in proximity to the composites, as studied previously. A part of these studies are materials placed in the sun to measure the change of surface temperature as a result of solar radiation and material properties, which effectively drives the geometric responsive changes. This study gives an indication of the relative energy build-up in respect to material surface characteristics. As the study is aimed for potential applications in both indoor and outdoor environments, measurements were conducted outside to understand the minimum relative difference between materials, due to the added cooling effect of the materials from air movement. The study was conducted on a flat horizontal roof, with isolation between materials and the roof surface, in Copenhagen on 25 May, with a semi-cloudy sky, ambient temperature of 21 degrees Celsius and wind velocity of $4 \mathrm{~m} / \mathrm{s}$, figure 2.

In another sub-study the bonding of materials into composites are examined, through the testing of five different bonding groups, with variations in tapes with core, tapes without core and primers. Additionally, five different approaches to inducing heat between layers are tested, including heated copper mesh $(0.5 \mathrm{~mm})$, aluminium mesh $(0.5 \mathrm{~mm})$, copper wire $(1 \mathrm{~mm})$, aluminium wire (1 $\mathrm{mm})$, kanthal wire (1 $\mathrm{mm})$ and heat tubing $(3 \mathrm{~mm})$. The internalised heat source is controlled by a microprocessor (MCU) setup, which enable coupling to sensor information. Thus, potentially allowing linking of environmental sensor data, via an MCU with embedding decision taking, to the actuation of the composite by inducing heat through the wire, creating a full 'system feedback' responsive system setup. Following these studies, extended tests to increase the impact of controlled heating by mesh/wire/tube, experiments with wrapping the composites were done. These studies include cotton, wool and cotton/aluminium, wool/aluminium covers, where the temperature build-up is measured and compared to non-covered composites, figure 3.

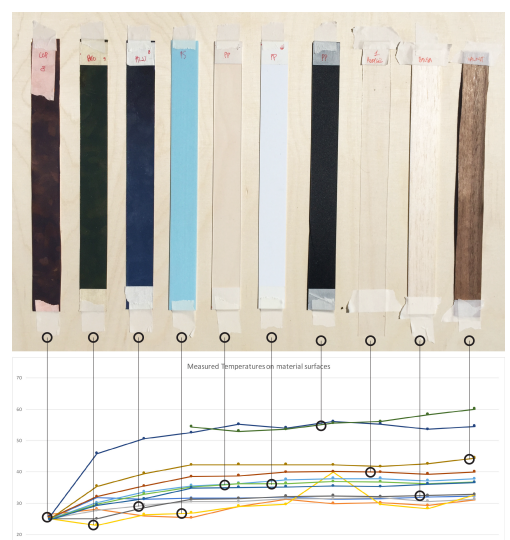



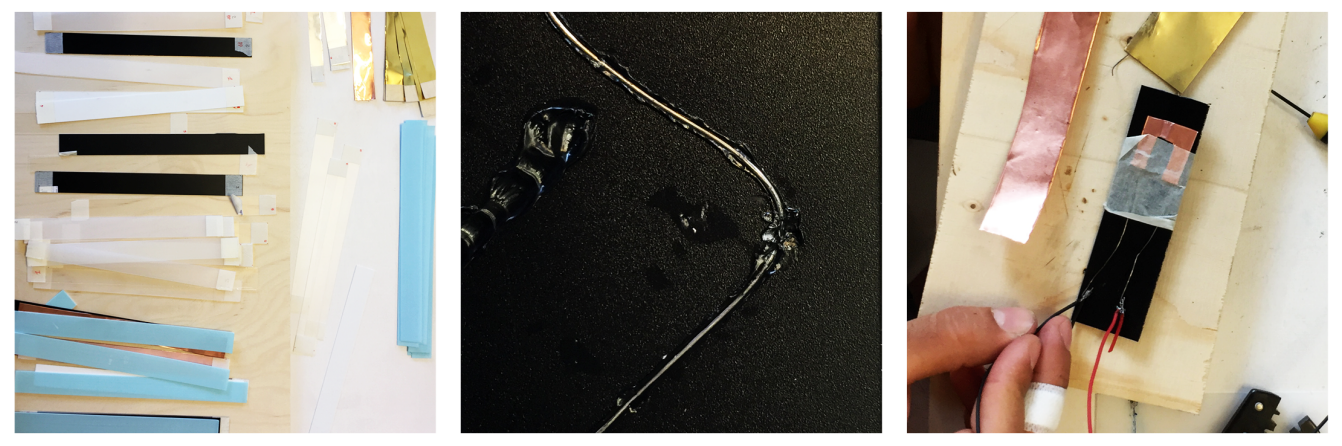

Figure 3

Prototyping studies for assessment of bending behaviour, energy transfer and energy

conservation of the tested composites.
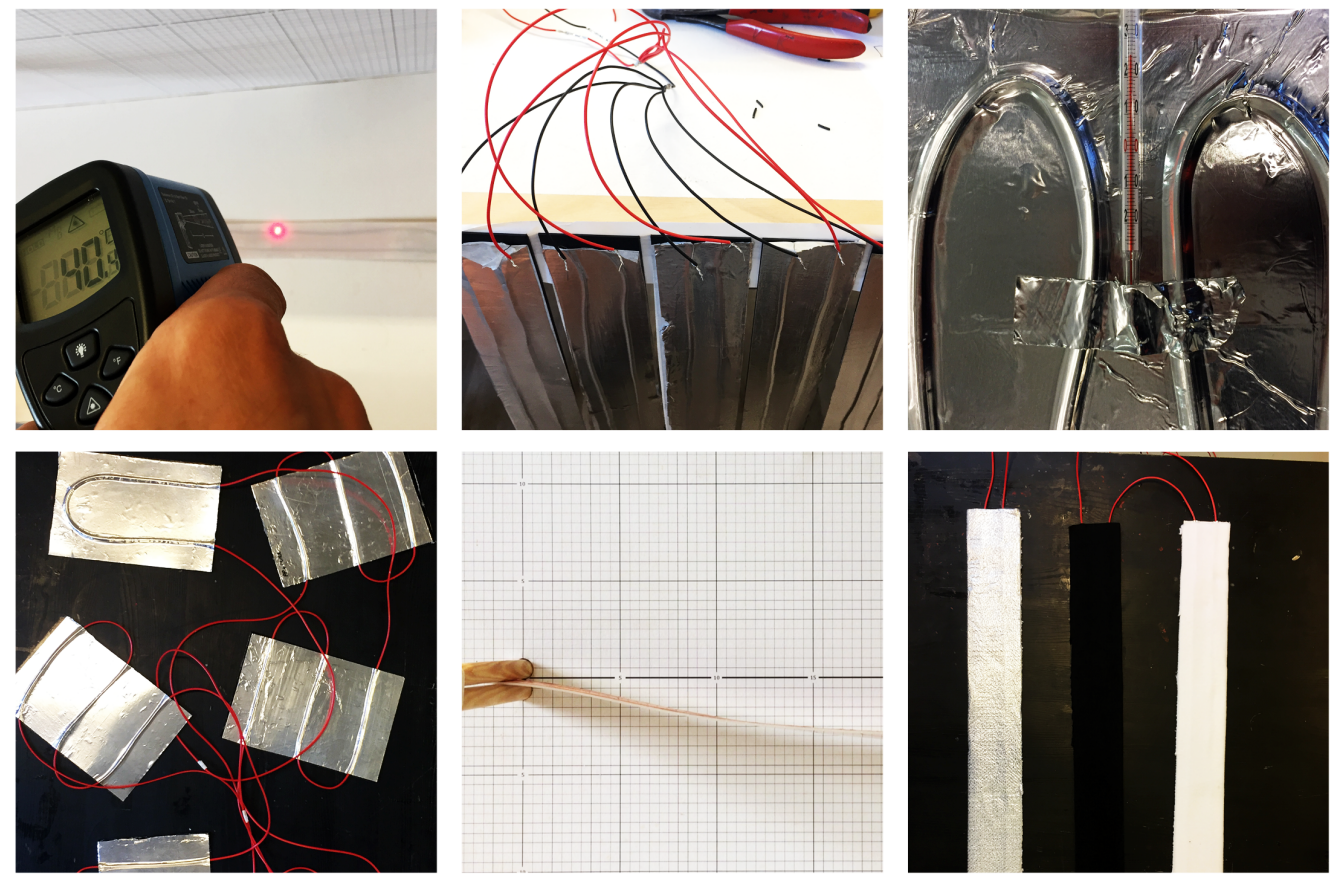


\section{DESIGN EXPERIMENTATION}

The experimentation with designing and making composites has been based on a project of creating a full scale $3 \times 10$ metre adaptive auxiliary building envelope. The focus of this paper, on the material studies for intrinsic driven responsive membranes, has been conducted in parallel to developments of a sensor-processing-actuation system and behavioural studies of the membrane-space-occupancy interactions. Due to the extent of the material studies and other aspects of the entire project, a full account of other aspects than the material studies are to be found in associated paper publications. Following the focus of the material studies in this paper, the experimentations described above where expanded to investigate cutting methods for the layer-assembled membranes. A series of material prototyping studies, as described above, revealed a high responsive bending performance and operational durability of the thin membrane $(1.5 \mathrm{~mm})$ when combining Oak $(0.5 \mathrm{~mm})$ and PE (1 mm). Oak has low thermal expansion parallel to the grain and PE has a high thermal expansion, as an isotropic material, in all directions. Combining materials with low and high energy surfaces require priming (3M Primer 94) the low energy surface to allow adhesion to the high energy surface through a non-core high bond adhesive 'tape' (3M VHB LSE.

The dimensions of the membrane responsive panels were developed through simulating the amount of solar energy passing through the composite membrane as a function of temperature variations, described and documented in the associated publication. The design experimentation of the material studies is then followed by investigating methods for cutting the generated membrane shapes, which ultimately becomes the material responsive elements of the envelope. For these studies, a Zund Eurolaser XL-1600 flatbed industrial machine is used with three different tool heads; a laser, a vertical oscillating knife, and, a 1-2 mm wide mill head. While the laser cuts through the composite (oak-glue-primer$\mathrm{PE})$, it melts the $\mathrm{PE}$, which then 'travels' towards the oak layer and creates edges and surface on the oak with unintended plastic coating. Using a knife method allows clean cuts, particularly along the grain of the oak, but fails to create a small gap, which is required when the composite attempts to bend out of its plane during thermal changes. Without the gap, friction is caused, reducing or prohibiting the bending action. Using a $1 \mathrm{~mm}$ drill head creates both a clean, non-melted and friction less result. Only drawback with this method is the accumulated PE on the drill head, due to heated material, which may cause reduced cutting ability, leading to mill head fracture, figure 5 . In addition, the application of a bonding adhesive without a core material, only including bonding material when rolled on, reduces fabrication problems, as only a minimum of material interfacing between the primary material layers are applied, figure 4.

Following the making of the composite panel, pattern cutting and integration into the mounting frames, onsite studies were conducted to understand and demonstrate the responsive performance of an exergy-based geometric bending of composite elements. The envelope panels are located on the inside of a glass pane, facing south-east, in a dense urban environment in Copenhagen. This means that the composites are effected by solar radiation on limited and specific hours of the day, that they are not cooled by air movement, and that they are embedded into an interior environment which ambient air is automatically kept at approximately 22 degrees Celsius from HVAC systems control. This means that the bending performance of the membrane is isolated to the solar radiation in relatively short and specified periods allowing a good reading of the solar radiation to material performance relations. As shown by the colouration/temperature studies above, dark materials have a significantly higher surface temperature, which increase response performance per degrees Celsius change. Hence, a black PE was used with the oak to form the primary material layers of the composite for the onsite demonstrator studies, figure 5 , 6,7 and 8 . 

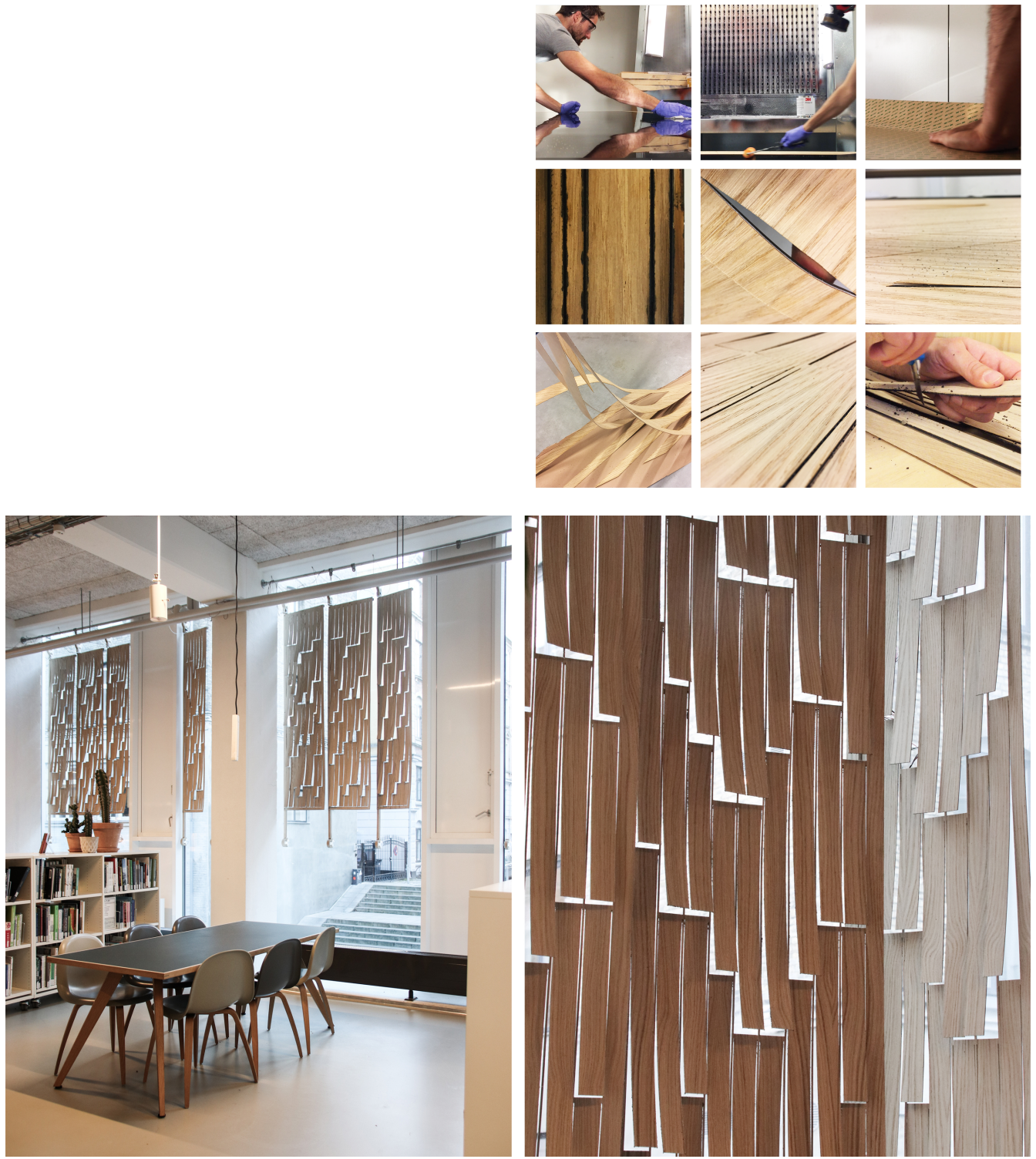

Figure 4

Testing of

membrane

fabrication

procedures to allow

material and

geometric

properties to be

maintained and

'released'

Figure 5

Full scale

demonstrator with

developed and

tested thermal

responsive

composites in the

Copenhagen

School of

Technology and

Design Library. Left

image shows the

developed

composite

mounted in

operable frames on

the glass pane.

Right side close-up

of the composite

bending and

creating light

permeability

through the

membrane. 
Figure 6

Dynamic bending of the developed composite after approximately 3 mins of direct solar radiation exposure.

Figure 7

Black PE side of the composite with orientation towards the solar energy source driving the exergy-based responsive change.

Figure 8

Temperature measurements on the installed demonstrator. The shaded oak surface side is warmed to 36 degrees Celsius through the composite, with the black PE surface side measured to 57 degrees Celsius where the ambient temperature is approximately 22 degrees Celsius.

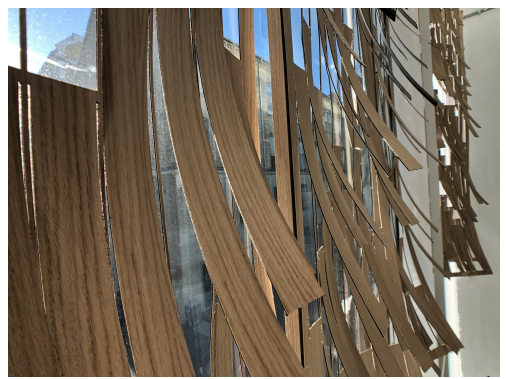

\section{RESULTS AND CONCLUSION}

The studies include a series of quantitative and qualitative results and conclusions.

- When solar radiation is the driver for actuation, colouration has a large impact on responsive performance. Comparative analysis measuring a range of materials show this, and measurements on the demonstrator support this, with the black PE side reaching 57 degrees Celsius, compared to the ambient temperature of 22 degrees Celsius. The specific degree of colour impact on performance is determined by the composite primary materials, the bonding structure, the geometric relations of size and thickness and the onsite explicit exposure to the solar energy.

- Coating the composites to increase thermal build-up by internal heating, significantly/completely reduces the ability to drive the actuation through the solar radiation as an exergy-based strategy. In addition, the coating adds weight to the active composite, which decrease response performance due to the extra load needed to be moved by the active composite. Hence, a coating/isolation approach to the responsive system must be taken when developing composites for the specific purpose.

- The composites developed hold some resistance to composite response behaviour fatigue. More than 150 days after fabrication, the composites are changing bending form and no delamination has been detected of the +400 bending elements. While the composites have not been tested in a climate laboratory where temperature constantly is changed up and down under regulated conditions, the composites have been placed in windows where they have been exposed to environmental conditions similar to possible applications in practice.

- The responsive operation of the composite is silent. This is favourable in spaces where en- 
vironmental sound levels are low, such as offices, study environments etc. Combined with the subtle and relative slow moment of the elements, the responsive membrane is perceived as a low-invasive active building layer.

- Given that the membrane is driven by solar environmental dynamics, it is limited to performance that are encoded into the membrane from its fabrication. Hence, such systems are not open to reprogramming of behaviour, which suggest the possibility of coupling to human and motorised systems, which may react to unforeseen or new conditions in a specific environment.

\section{DISCUSSION}

The presented studies contribute to the understanding and application of material driven responsive performance membranes, through a large material study and resultant catalogue of composite behaviours. It also illustrates how these can be bonded and cut into specified membranes reacting from thermal changes in an environment. The study also finds that additional activation of the responsive capacities through inducing heat between layers and insulating the composite to improve and maintain heat build-up are problematic. This is also due to the wire reducing bonding between layers, resisting bending (depending on wire layout). Further studies into internal heating and regulating of stimuli for responsive behaviour may however change the instrumentality of the responsive material system into a more open and adaptive operated system based on unknown conditions when the envelope is fabricated. Hence, this may be an option for controlling bending through an MCU with embedded behaviour controls, yet, enhancements of this approach through insulation of the composites also means isolation from the thermal microclimatic environment which activate responsive behaviour without applying electrical energy. For this reason, the study points to coupled, or hybrid, systems, where intrinsic material-driven response behaviour is paired with another response system, such as proposed in the general project, allowing material-driven and MCUdriven systems to operate in combination without limiting the capacities of each their specific strengths as environmental adaptive mechanisms for architectural envelopes.

\section{ACKNOWLEDGEMENT}

The authors would like to thank the COWI Foundation for supporting the research.

\section{REFERENCES}

Correa, D, Krieg, D, Menges, A, Reichert, S and Rinderspacher, K 2013 'HygroSkin: A prototype project for the development of a constructional and climate responsive architectural system based on the elastic and hygroscopic properties of wood', ACADIA 13: Adaptive Architecture [Proceedings of the 33rd Annual Conference of the Association for Computer Aided Design in Architecture (ACADIA) ISBN 978-1-926724-225] Cambridge 24-26 October, 2013), pp. 33-42

Foged, IW and Pasold, A 2010 'Performative Responsive Architecture Powered by Climate', ACADIA 10: LIFE in:formation, On Responsive Information and Variations in Architecture [Proceedings of the 30th Annual Conference of the Association for Computer Aided Design in Architecture (ACADIA) ISBN 978-1-4507-34714] New York 21-24 October, 2010), pp. 243-249

Foged, IW and Pasold, A 2015 'Development of a Method and Model for Programming Material Behaviour in a Responsive Envelope', Martens, B, Wurzer, G, Grasl T, Lorenz, WE and Schaffranek, R (eds.), Real Time - Proceedings of the 33rd eCAADe Conference - Volume 2, Vienna University of Technology, Vienna, Austria, 1618 September 2015, pp. 449-458

Foged, IW and Pasold, A 2016 'An Oak Composite Thermal Dynamic Envelope', Structures and Architecture Beyond their Limits : 3rd International Conference on Structures and Architecture Conference Proceedings. Taylor \& Francis

Kanthal, AB 2008, Thermostatic Bimetal Handbook, Kanthal $A B$

Reichert, S, Menges, A and Correa, D 2015, 'Meteorosensitive architecture: Biomimetic building skins based on materially embedded and hygroscopically enabled responsiveness', Journal of Computer-Aided Design, 50, pp. 50-69 